\title{
MASS CONSERVATION AND RAPID MASS LOSS \\ ON THE MAIN SEQUENCE
}

\author{
T.J. Mazurek \\ Department of Astronomy \\ University of Texas at Austin
}

The rapid mass loss observed in 0 stars can affect their evolution dramatically if it takes place during core hydrogen burning on the main sequence. Conti has suggested ${ }^{1}$ that this is the case for the brightest of stars, and that these stars evolve into Wolf-Rayet (WR) stars. Support for this scenario comes from the similarityl in the spectra of the Of and WR stars, and from the observed helium enrichment of Wolf-Rayet WN stars along with the correlation ${ }^{2}$ of the latter with the most luminous 0 stars. If the most massive of stars evolve to WN stars of relatively low mass, one needs to determine the range of zero-age stellar masses where such an evolution occurs. This communication abstracts some observational evidence that bears on the question of the minimum zero-age mass for rapid mass loss on the main sequence. It then summarizes the author's investigation of a model for mass loss where photospheric acoustic waves control the flow rate.

The HR-diagram of stellar models ${ }^{3}$ can be used to estimate ${ }^{1}$ the masses of 0 stars. Figure 1 shows an HR-diagram for observed massive stars 4 and the theoretical main sequence strip. Only luminosity classes IV and $V$ and f-type stars are included. It is evident from the figure that the $\mathrm{f}$-characteristics appear in 0 stars with $\mathrm{M} \approx 30 \mathrm{M}_{\Theta}$. Of stars are present along the zero-age line, and the dense envelopes associated with these stars can appear in unevolved stars.

Empirical determinations of rates of mass loss are available for $\zeta$ Pup $\left(\sim 7 \times 10^{-6} \mathrm{M}_{\odot} \mathrm{yr}^{-1}\right.$, ref.5) and $\tau$ Sco $\left(\sim 7 \times 10^{-9} \mathrm{M}_{\odot} \mathrm{yr}^{-1}\right.$, ref. 6). In attempts to correlate empirically determined rates with theoretical ste1lar models only stars near the zero-age line should be used. For rapid mass loss, the internal structures of evolved stars is not known since the behavior of mass loss rates as evolution progresses is poorly determined. Fortunately $\zeta$ Pup and $\tau$ Sco fall near the zero-age line in the HR-diagram, and presumably have structures similar to the theoretical 3 ones. Their positions in the HR-diagram indicate masses of $\sim 100 \mathrm{M}_{\odot}$ and $18 \mathrm{M}_{\Theta}$ for $\zeta$ Pup and $\tau$ Sco, respectively. 


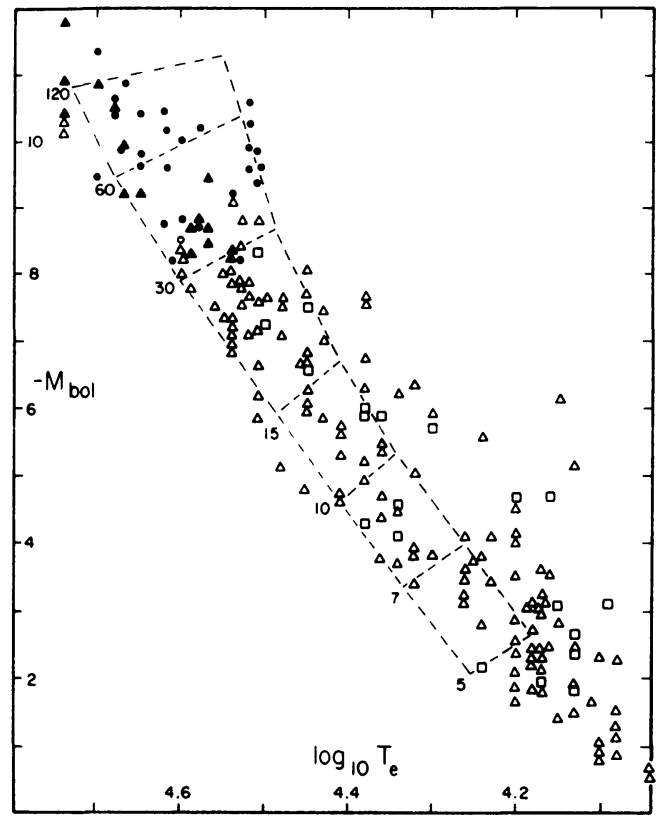

Fig. 1.--The HR-diagram (masses along ZAMS in $\mathrm{M}_{\Theta}$ ). Luminosity classes IV, $\mathrm{V}$, and other are denoted by $\square, \Delta$, and 0 , respectively; f-type stars have filled symbols.
The proposed mechanisms for powering stellar winds have been discussed 7 extensively at this symposium. The presence of a radiation driven mechanism apparently is required to achieve the high terminal velocities that are observed. But the spectroscopically inferred presence of ions having a large range of ionization potentials indicates temperatures much greater than those expected if radiative acceleration is the sole mechanism. This implies that another wind mechanism is present. The apparent $1 \mathrm{ack}^{8}$ of correlation between observed terminal velocities and stellar luminosities may indicate that radiation forces can act efficiently to expel all matter that is ejected from the stellar surface. Hence a non-radiative mechanism may actually control the rate of mass loss, while radiation pressure completes the acceleration of the wind to its terminal velocity.

A coronal model for the wind flow near the stellar surface has been presented by Hearn ${ }^{9}$. The sources of the energy required to maintain the corona need to be examined in

detail. If surface turbulence is present in 0 stars, it will generate sound waves which can deliver energy to the corona. The presence of macroturbulence would explain in a straight forward manner the observed dearth of 0 stars with sharp lines 10 . The process producing the stellar wind may thus begin with sound waves generated in the photosphere, which then steepen into shocks giving a hot corona, and radiation forces supply the final acceleration to the flow.

The connection between acoustic wave generation and coronal rates of mass loss has been examined in detail for main sequence stars by the author. In this investigation the velocities of the surface turbulence are determined by photospheric conditions which are directly related to stellar luminosities, temperatures, and masses. Theories ${ }^{11}$ of acoustic wave generation in turbulence are applied to determine energy fluxes from the photospheres. The energy balance between acoustic input and coronal losses is then used to determine the coronal structure, and hence the rate of mass flow. Published theoretical models ${ }^{3}$ are then used to estimate rates of coronal flow on the main sequence. Some salient results of this investigation are the following. 
The predicted turbulent velocities at the photosphere range between

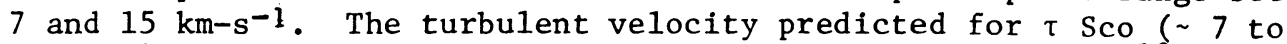
$9 \mathrm{~km}^{-1} \mathrm{~s}^{-1}$ ) is in good agreement with empirical determinations ${ }^{12}$. The variation in velocity over the different spectral classes is consistent with the lower envelope 10 to the Doppler broadening of observed 0 stars. A considerable increase in the rate of acoustic energy generation above that given by Stein 11 is necessary to produce mass flow rates as high as those of $\zeta$ Pup. Turbulent magnetic fields in the photospheres can give the enhancement required. With enhanced acoustic generation, the coronal mass flow rates predicted for $\zeta$ Pup and $\tau$ Sco $\left(\sim 6 \times 10^{-6}\right.$ and $12 \times 10^{-9}$ $\mathrm{M}_{\ominus} \mathrm{yr}^{-1}$ ) are in good agreement with the empirical values quoted above.

For stars with $M \gtrsim 30 \mathrm{M}_{\Theta}$, the radiative losses outside the corona's critical point are so large that the coronal flow in that region becomes energetically inconsistent. Radiative acceleration becomes essential for mass 1oss. The high cooling may give a density increase in the wind flow, and hence be the cause of the observed high densities in of stars. For stars with $M \geq 60 \mathrm{M}_{\Theta}$, the rate of mass flow increases rapidly with evolution across the main sequence strip. A runaway in the rates of mass loss is indicated. These stars should then evolve into WN stars. The lower mass limit for this behavior is consistent with an empirical estimate ${ }^{2}$. The stars with $30 \mathrm{M}_{\Theta} \leqslant \mathrm{M} \leqslant 60 \mathrm{M}_{\Theta}$ are predicted to lose only a small fraction ( $s 10 \%$ ) of their mass during core hydrogen burning.

This work was supported in part by NSF (Grant No. AST-76-07629).

1. Conti, P.S. 1976, Mem. Soc. Roy. Sci. Liége, 9, 193.

2. Moffat, A.F.J., and Seggewiss, W. 1979, preprint.

3. Stothers, R. 1972, Astrophys. J., 175, 431.

4. For most stars, $\mathrm{M}_{\mathrm{bol}}$ and $\mathrm{T}_{\mathrm{e}}$ were determined from the $(B-\mathrm{V})$ given by G.L.H. Harris (1976, Astrophys. J. Supp1., 30, 451) with the conversions of A.D. Code, J. Davis, R.C. Bless and R.H. Brown (1976, Astrophys. J., 203, 417) and D.C. Morton (1969, Astrophys. J., 158, 629). For some stars the results of ref. 8 or those of P.S. Conti and M.L. Burnichon (1975, Astron. Astrophys., 38, 467) were used.

5. Lamers, H.J.G.L.M., and Morton, D.C. 1976, Astrophys. J., 32, 715.

6. Lamers, H.J.G.L.M., and Rogerson, J.B. 1978, Astron. Astrophys., 66,417 .

7. See in particular the contributions of J. Cassinelli, J. Castor, A.G. Hearn, H. Lamers, and R. Thomas.

8. Snow, T.P., and Morton, D.C. 1976, Astrophys. J. Supp1., 32, 429.

9. Hearn, A.G. 1975, Astron. Astrophys., 40, 277, and 40, 355.

10. Conti, P.S. and Ebbets, D. 1977, Astrophys. J., 213, 438.

11. Stein, R.G. 1968, Astrophys. J., 154, 297; and Kulsrud, R.M. 1955, Astrophys. J., 121, 461.

12. Smith, M.A., and Karp, A.H. 1978, Astrophys. J., 219, 522. 